\title{
Weaving tangled webs
}

\author{
From the realization of their true nature one hundred years ago to the latest approaches for structuring materials \\ using molecular weaving, high-molecular-weight polymers still have much to offer society.
}

$\mathrm{P}$

olymers are present throughout society in various applications, such as packaging, electronics and medical applications. Depending on the structure and molecular architecture, which can be controlled at synthesis by the chemist, different properties can be engineered in materials such as polystyrene, polyester, polyvinyl chloride or polyamides. This year marks the 100th anniversary of the finding that polymers are formed from linking monomers together by the formation of covalent bonds ${ }^{1}$, and so paving the way to their current ubiquity.

In retrospect, the original nineteenth-century idea that polymers are compounds with the same chemical composition but different properties, such as benzene $\left(\mathrm{C}_{6} \mathrm{H}_{6}\right)$ and styrene $\left(\mathrm{C}_{8} \mathrm{H}_{8}\right)$, seems unfortunate ${ }^{2}$. Only in 1922 did Hermann Staudinger provide the first experimental evidence for long, covalently bound polymer chains by hydrogenating natural rubber. $\mathrm{He}$ and his co-workers observed that hydrogenation could proceed without forming volatile low-molecular-weight cyclic hydrocarbons, implying high-molecular-weight polymers tangle to form the rubber instead. It is fortunate indeed that Staudinger ignored his colleagues' advice on this 'grease chemistry' and focused his research on polymer chemistry, which was recognized by the Nobel Committee for Chemistry in 1953, and which has led to other breakthroughs that continue to impact modern life.

Indeed, research into polymer applications is still ongoing. Semiconducting polymers can be used to form flexible electronic devices that are lightweight and easily tunable ${ }^{3}$. Polymers can act as solid electrolytes to enable Li-ion transport from cathode to anode in battery systems, due to their enhanced electrochemical stability and elasticity; compared with the products that form during cycling cathode materials that operate at higher capacity and voltage can be stabilized $^{4}$. And by the appropriate choice of monomer structure, polymer chain packing can be tuned to modulate the interchain space, allowing the selective separation of gases, and enabling membranes that could be used for industrially relevant separations of gases such as $\mathrm{CO}_{2} / \mathrm{N}_{2}\left(\right.$ ref. ${ }^{5}$ ).

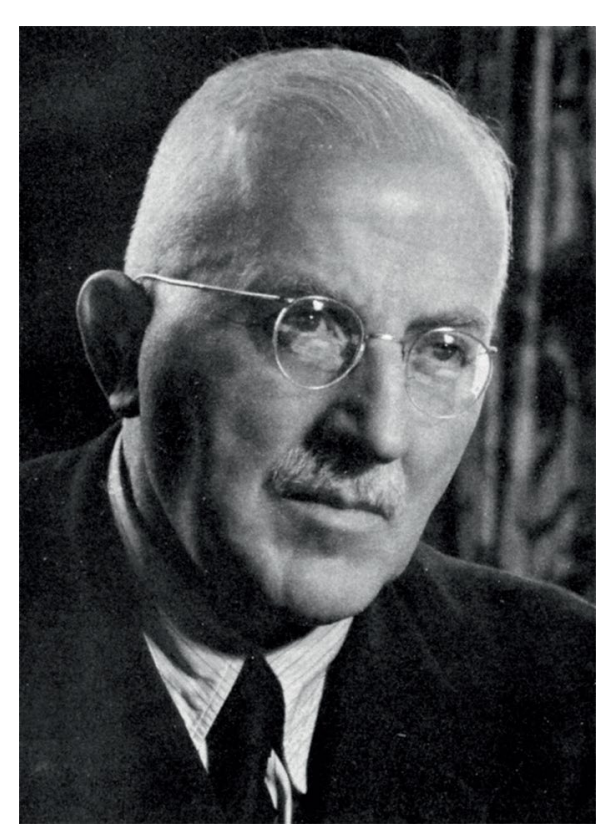

Photo of Hermann Staudinger. Credit: Matteo Omied / Alamy Stock Photo.

For all of these examples, and many others, the control of structure is key. Here, it is possible to connect the latest advances in polymer assembly with some of the oldest technologies in the human toolkit; weaving strands in order to generate long-range order. In a Perspective published in this issue of Nature Materials, David Leigh, Liang Zhang and colleagues overview the history, mechanisms, strategies and challenges of weaving at the molecular scale. The concept of molecular weaving is to regularly entangle one-dimensional (1D) molecules into extended 2D or 3D networks, which may present different properties. As the authors note, the initial idea that robust molecular weaves can only be formed from covalently linked and entangled molecular strands may not be appropriate. Non-covalent bonds might be strong enough to restrain strands while reversible covalent bonding might result in dynamically labile rather than positionally confined structures. Insights from molecular knots could be of value in learning how to weave molecular strands. Various models of mechanically restricted systems are considered, such as tube, slip-link or slip-spring models. These models, as well as insights from topology, could, in turn, help in the understanding of how weaving modifies polymer properties.

The authors also survey the different strategies that can be used to form molecularly woven organic polymers. They note that covalent organic frameworks can be woven in three dimensions, with the resulting weave showing increased elasticity after further treatment. Metal-organic frameworks can be used as templates for the formation of 2D hydrogen-bonded frameworks, while supramolecular kagome meshes can be weaved. In both cases, the weave is bound by dynamic non-covalent bonding, although it is speculated that modification to enable covalent bonding could be used to kinetically lock strand entanglement. Weaves can also be generated by tessellation of prewoven tangles; following ion-template removal the weave was almost as twice as strong as the individual molecular strands. Key challenges are outlined, including is it possible to form different weave patterns using the same molecular strands? How do weaving patterns and interstrand interactions affect properties? And how can the mesh of the weave be exploited for additional functionality by controlling size, shape or chemical functionality? The authors also query whether it would be possible to weave preformed commercial polymers such as polystyrene, which they envisage may require artificial molecular chaperones.

The processability and tunability of polymers has enabled property control for many commercial applications. With increasing insight and control of their structure, both experimentally and computationally ${ }^{6}$, better control of their properties as well as new applications will surely be achieved.

Published online: 3 March 2022 https://doi.org/10.1038/s41563-022-01219-z

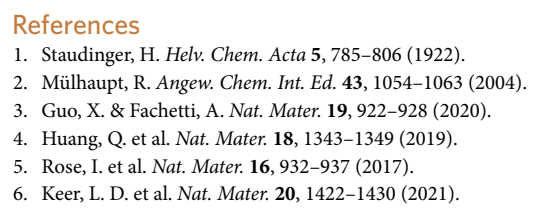

